\title{
Problems and monitoring the spread of the ecologically dangerous plant Heracleum Sosnowskyi in urbanized areas and methods to combat it
}

\author{
Vladimir Bogdanov ${ }^{1}$, Aleksey Osipov ${ }^{2}$, Vitaly Garmanov ${ }^{1}$, Galina Efimova $^{1}$, Alexander \\ Grik $^{1}$, Boris Zavarin ${ }^{1}$, Vitaly Terleev ${ }^{3,4}$, and Aleksandr Nikonorov ${ }^{5,{ }^{*}}$ \\ ${ }^{1}$ St.Petersburg State Agrarian University, Peterburgskoe shosse, 2, St. Petersburg-Pushkin, 196601, \\ Russia \\ ${ }^{2}$ A.F. Mozhaysky's Military-Space Academy, Zhdanovskaya Naberezhnaya, 13, St. Petersburg, \\ 197198, Russia \\ ${ }^{3}$ Peter the Great St.Petersburg Polytechnic University, Polytechnicheskaya, 29, St. Petersburg, \\ 195251, Russia \\ ${ }^{4}$ Agrophysical Research Institute, Grazhdansky pr., 14, St. Petersburg, 195220, Russia \\ ${ }^{5}$ LLC «Gazprom pererabotka Blagoveshchensk», p. Gazoprovod, p. Sosenskoe, Moscow, 108814, \\ Russia
}

\begin{abstract}
The article contains information on the breeding of Heracleum Sosnowskyi for cultivation as a forage crop in the post-Soviet period. Information about the ecological danger of the spread of this plant is given. Its juice contains furocoumarins, which, when exposed to human skin under the influence of ultraviolet rays, cause serious burns on the surface of the body. The article presents materials testifying to the increased adaptability of the Heracleum Sosnowskyi to the habitat conditions and its high fertility. The spread of Heracleum Sosnowskyi in urban areas (in parks, playgrounds on undeveloped lands, etc.), as well as in rural settlements of the country, due to the environmental hazard for people, leads to the removal of land from economic circulation. The authors of the article have established the regions and reasons for the spread of Heracleum Sosnowskyi in the European part of Russia. The substantiation of the monitoring of lands for the registration of habitats of this dangerous for the ecosystem is given. The article provides information on assessing the extent of the spread of Heracleum Sosnowskyi in the territory using remote sensing using unmanned aerial vehicles. The article discusses measures to combat Heracleum Sosnowskyi.
\end{abstract}

\section{Introduction}

In the late 40's of last century, breeders bred varieties of Heracleum Sosnowskyi called "Severyanin". It began to be introduced everywhere in agriculture. Since the 1990s, in the

\footnotetext{
*Corresponding author: coolhabit@yandex.ru
} 
conditions of economic and land reforms, the use of Heracleum Sosnowskyi has been abandoned [1-4].

During work planning to combat Heracleum Sosnowskyi, it is important to know the territorial distribution of the littered lands, their area and the degree of clogging. To solve this problem, it is necessary to monitor the land according to remote sensing. The authors based the study on the following hypothesis: if a method for monitoring soils contaminated with Heracleum Sosnowskyi is developed using remote sensing data, the quality and efficiency of the plan will be improved.

More than 20 species of various species of the Heracleum plant are known in Europe. Three species, Heracleum Mantegazzianum, Heracleum Sosnowskyi, and Heracleum Persicum, make up a group of plants known as giant Heracleum. Heracleum Sosnowskyi was identified in 1944 by I.P. Mandenova and named in honor of the famous researcher of the flora of the Caucasus D.I.Sosnovsky. His homeland is the Central and Eastern Caucasus, Transcaucasia and Turkey. Alien invasive species introduced into the region from other countries can grow in a new place for decades without any further spread. When a species has reached an exponential phase, its spread becomes rapid and difficult to control. This happened with the giant Heracleum Sosnowskyi in Europe and the European part of Russia. Due to the fact that Heracleum Sosnowskyi has a significant green mass and contains a large amount of protein (10-17\% crude protein), this plant was taken for selection in order to obtain feed for animal husbandry. Since the 1960s, it has been cultivated as a fodder plant for feeding livestock in agricultural enterprises in many regions of Russia: in the Leningrad, Moscow, Ivanovo, Kirov, Pskov regions, in the Komi Republic, as well as in Belarus and the Baltic countries. In the early 90s, due to the economic and land reform in our country and the collapse of state and collective farms, it got out of control and began to intensively disperse on abandoned lands, in horticultural areas, on the slopes of reclamation canals, roadsides, and more recently time began to penetrate the lands of settlements. Heracleum Sosnowskyi juice contains furocoumarins when they get on human skin under the influence of ultraviolet rays, it leads to severe burns on the body surface. In some cases, Heracleum Sosnowskyi juice can cause toxic poisoning in humans, which is accompanied by disruption of the nervous system and heart muscle. Therefore, this ecologically dangerous plant poses a serious threat to human health. In 2015, Heracleum Sosnowskyi was included in the sectoral classification of weeds of the Russian Federation under number 5506 [2]. This legal framework made it possible to take measures to combat it.

\section{Material and methods}

The aim of the study was to develop a methodology for monitoring land clogging by Heracleum Sosnowskyi according to remote sensing of the territory using the NDVI (Normalized Difference Vegetation Index) and ClGreen (GreenChlorophyllIndex) indices. The initial materials in the study were field observations of the distribution of Heracleum Sosnowskyi within the model territory, remote sensing data obtained using an unmanned aerial vehicle in flight. Due to the fact that the time series of NDVI during the intervegetation period do not provide a clear phenological division, therefore, the raids were performed during the vegetation period [5]. The NDVI vegetation index characterizes the difference between the red (RED) and near infrared (NIR) parts of the electromagnetic spectrum, which could be calculated.

The purpose of the study was to study the problem of dispersal on the territory the environmentally dangerous plant - Heracleum Sosnowskyi and to develop monitoring technology for establishing its habitat based on remote sensing.

The object of study are the Heracleum Sosnowskyi and areas overgrown with this plant. 
Heracleum Sosnowskyi can form plant colonies of various densities ranging from a few square meters to several hectares. It has almost no natural enemies. Due to the presence of a poisonous substance - furocumarin in its leaves and stem, it is little damaged by herbivores. Heracleum Sosnowskyi is resistant to unfavourable climatic conditions, has no competitors, actively suppresses the growth of other species of plants. This plant captures better lit areas and more fertile soils.

Heracleum Sosnowskyi has an extremely high reproductive capacity and aggressiveness towards native plant species, as well as ecological valence (the degree of adaptability of a living organism to changes in environmental conditions), which allows it to freely capture new territories, forming monoid communities. Depending on the growing conditions, one Heracleum Sosnowskyi plant is able to produce between 4 and 20 thousand seeds. Seeds are tied not only by cross-pollination, but also by self-pollination. Thus, one plant after fructification can give rise to a new population. It can completely occupy the existem, displacing natural vegetation. The dispersal of Heracleum Sosnowskyi on the territory leads to the withdrawal of agricultural land from economic circulation, forms a mono-dominant phytocenosis, which harms the environment and poses a danger to people.

The shape of the Heracleum Sosnowskyi seeds (samara) allows to transfer themselves from the plant 's growth sites over long distances. The main sources of seed transfer are car wheels, rivers and reclamation channels, birds, wind, and human clothing.

Studies of natural and climatic conditions of the Russian European part regions and the zone of Heracleum Sosnowskyi dispersal showed that the Heracleum Sosnowskyi growth territories are characterized by an average annual excess of precipitation over evaporation.

\section{Results and discussions}

As research shows, Heracleum Sosnowskyi can form colonies of plants of various densities from several square meters to several hectares. He has almost no natural enemies. The Heracleum Sosnowskyi is resistant to unfavorable climatic conditions, has no competitors, actively inhibits the growth of other plant species. This plant captures better illuminated areas and more fertile soils.

The Heracleum Sosnowskyi has an extremely high reproductive ability and aggressiveness towards native plant species, as well as ecological valence (the degree of adaptability of a living organism to changes in environmental conditions), which allows it to freely capture new territories, forming monospecific communities.

The shape of the seeds of the Heracleum Sosnowskyi allows it to be transferred from the plant's growing areas over long distances. The main sources of seed transport are: seedclogged soil, car wheels, rivers and drainage canals, birds, wind and human clothing.

Vologda, Leningrad, Moscow, Novgorod, Pskov, Tula, Yaroslavl, Tver, Nizhny Novgorod and other regions [2] were subjected to a massive invasion of Heracleum Sosnowskyi. A serious situation has developed in the Komi Republic: the plant communities of Heracleum Sosnowskyi occupy vast territories in the southern part of the republic. Large areas in the countries of Latvia, Estonia, Ukraine and Belarus are littered with this pest.

Studies of the natural and climatic conditions of the regions of the European part of Russia and the distribution zone of Heracleum Sosnowskyi have shown that the growing areas of hogweed are characterized by an average annual excess of precipitation over evaporation.

One of the most affected by the invasion of the Heracleum Sosnowskyi is the Leningrad region. The ecological conditions in the region are favorable for the reproduction of this plant. Before the introduction of measures to combat it, the number Heracleum Sosnowskyi in the region was more than 30 thousand hectares. 
Currently, the problem of the spread of Heracleum Sosnowskyi has gone beyond the boundaries of agricultural lands. This aggressive plant began to spread intensively in settlements and in urban areas. In the cities, Heracleum Sosnowskyi began to populate undeveloped territories, by bringing seeds by the wind, with the wheels of cars, and birds. On playgrounds, lawns, he began to appear in connection with the delivery of soil contaminated with Heracleum Sosnowskyi seeds to these urban objects from other places. (Fig. 1). Therefore, the problem of the spread of an ecologically dangerous plant - the Heracleum Sosnowskyi in settlements has become quite acute, which must be addressed first of all.

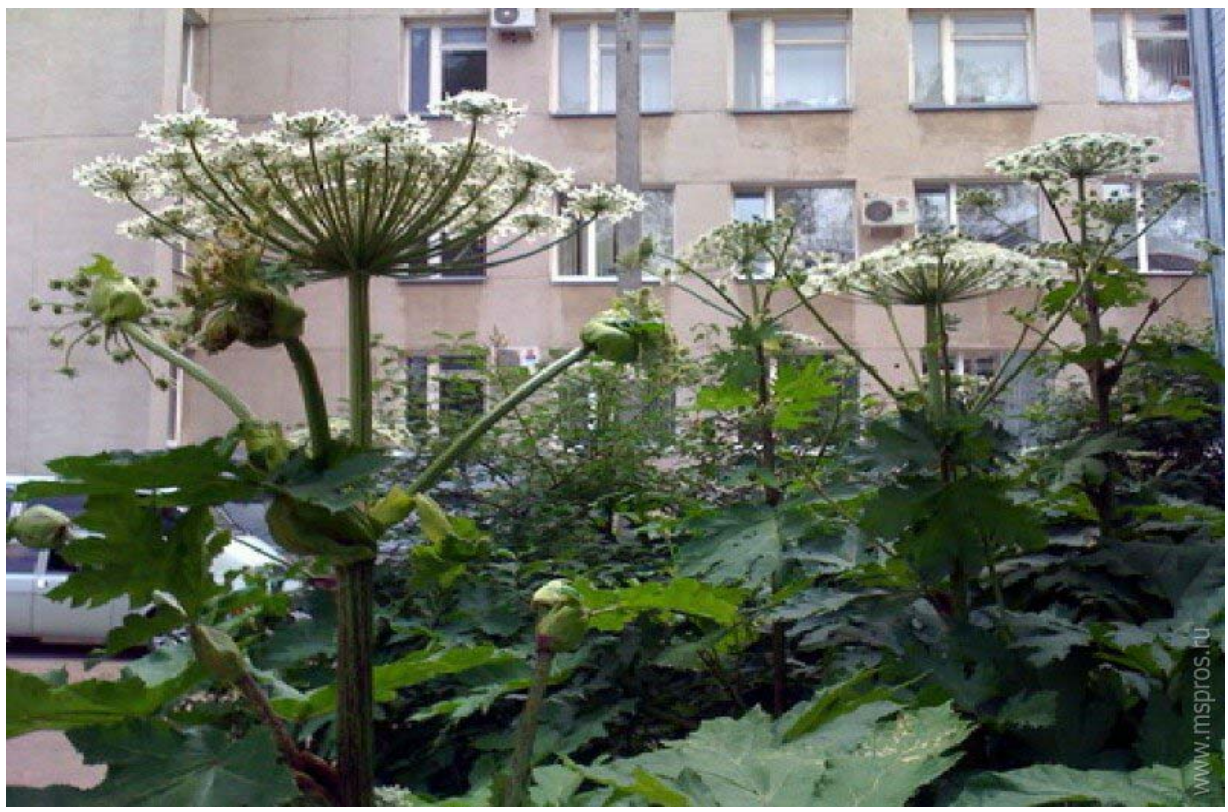

Fig. 1. Distribution of Heracleum Sosnowskyi in the area near residential buildings.

The spreading rate of Heracleum Sosnowskyi in the area with sowing of perennial grasses (as an option for lawns) was studied in the field. Our research, carried out in a meadow in the Leningrad region, made it possible to establish the stages of distribution of Heracleum Sosnowskyi in the meadow. The source of the clogging was the waters of the reclaimed canal with the presence of cow parsnip seeds in it and the wind. First, the first borscht plants appeared on the slopes of the reclamation channel. After 3 years, he occupied the territory along the main canal in a continuous strip at a distance of 10-15 meters from the canal. Then, every year, he began to intensively settle in the meadow. (Fig. 2). Every year Heracleum Sosnowskyi captures up to $5 \%$ or more new territories in the zone of its settlement in the Leningrad region. To plan the fight against Heracleum Sosnowskyi, it is important to know the area and degree of contamination by the Heracleum Sosnowskyi farmland, to determine the boundaries of the contaminated hogweed, to identify the dynamics and forecast of the spread of this ecologically dangerous plant. 


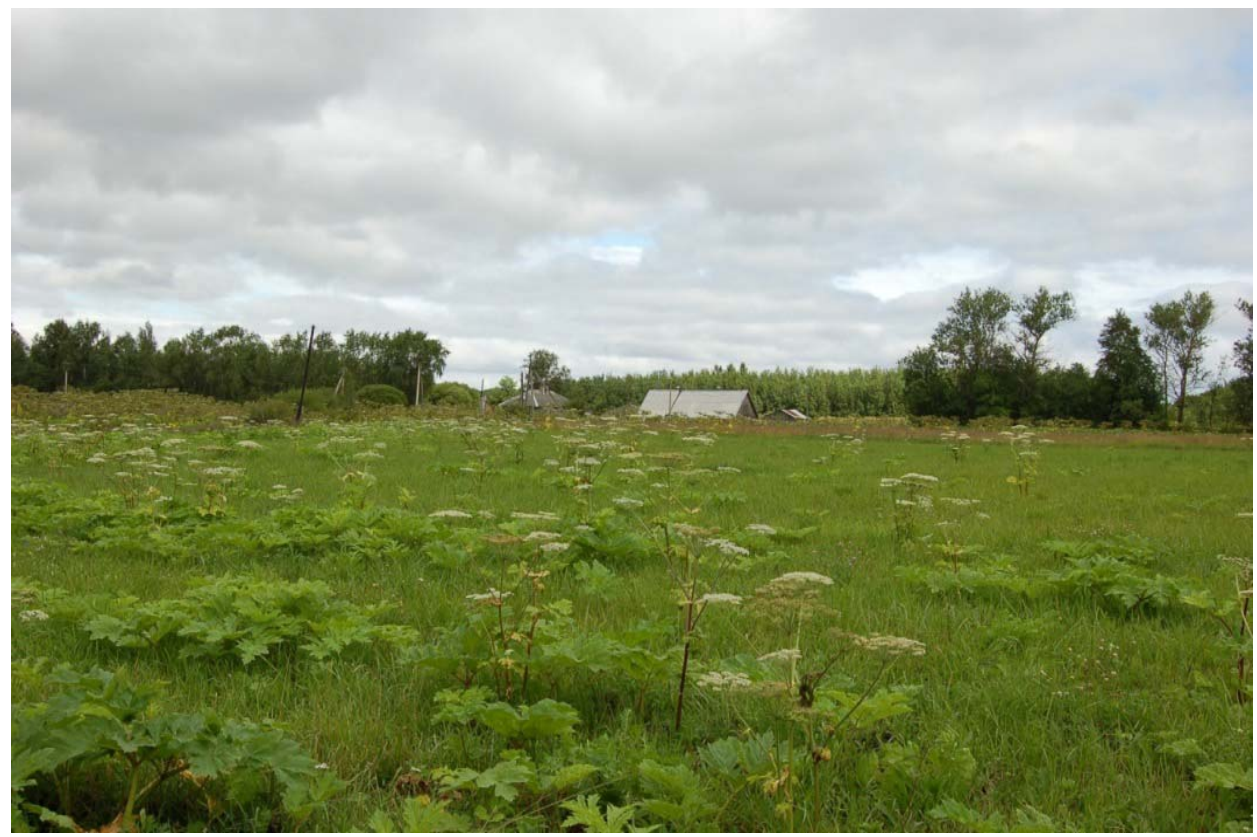

Fig. 2. Settlement of the meadow with Heracleum Sosnowskyi.

This requires monitoring of lands and subsequent mapping of the habitats of the Heracleum Sosnowskyi. Modern technologies of remote sensing of the earth (ERS) make it possible to carry out such effective monitoring and obtain information about the state of land resources without direct contact with the object under study. Specialized PC technology provides spectral analysis and registration of differences in reflected sunlight from the earth's surface covered by the studied plants in a state at different stages of their development. The brightness values are combined into certain channels that are informative for highlighting the object under study. They are used to calculate "spectral index" values. Based on these data, an image is constructed that corresponds to a certain value of the index in each pixel, which allows you to select the object under study and assess its state $[3,4]$. In this regard, special field studies are required to obtain spectral characteristics of the physiological state of plants (boletus Sosnowskyi), which are obtained on the basis of the developed vegetation indicators [5]. Spectral characteristics of the physiological state of plants make it possible to determine the parameters necessary for weed control: phases of plant development

In order to study the effect of chemical preparations (herbicides) on the reproductive ability and destruction of Heracleum Sosnowskyi, a field experiment was laid in the Vyborg district of the Leningrad region. The experiment tested the effect of a mixture of herbicides - glyphosate and Banvela BP - in doses from 1 to 8 liters per $1 \mathrm{~m} 2$ of soil surface. The processing of Heracleum Sosnowskyi plants was carried out in the stalking phase on June 9. The research results showed that the most effective dose for the destruction of Heracleum Sosnowskyi was a mixture of glyphosate and Banvela BP at a dose of $8 \mathrm{~g} / \mathrm{m} 2$ in a ratio of 3: 1, respectively. 3 weeks after the treatment, the stems and leaves of the Heracleum Sosnowskyi wilted, and the roots of the first, second and third order turned black and lost the ability to renew the vegetative mass. However, the seeds of Heracleum Sosnowskyi, which were in the soil, did not suffer and after a while they sprouted. One month after the chemical treatment of the Heracleum Sosnowskyi, the ecosystem began to gradually recover from the forbs. In addition to studying the above techniques that contribute to the destruction of Heracleum Sosnowskyi, in the territory of 
the settlement, which was largely infested with Heracleum Sosnowskyi (on average, 2 plants per $1 \mathrm{~m} 2$ ), the meadow surface was mulched with a black film. The sheathing of the phytocenosis infested with Heracleum Sosnowskyi with a film was carried out in early May, when the plants of the Heracleum Sosnowskyi were in the rosette phase. After 3.5 months, the film was removed. The result of this technique was $100 \%$ death of Heracleum Sosnowskyi plants. In addition, studies of the seeds of this plant, collected from the soil surface to assess their viability after mulching, showed a complete loss of their germination. Thus, the method of mulching Heracleum Sosnowskyi in the early stages of its development is an effective method for combating this plant. Further tillage and sowing of perennial grasses helps to restore the natural ecosystem. Agrotechnical methods of dealing with hogweed, such as mowing hogweed plants before seeding the plants and digging up its root system, were also effective. Currently, new technology is being used to combat Heracleum Sosnowskyi. After remote sensing of the territory, they use unmanned equipment - drones. The drone tank is filled with herbicides to treat areas contaminated with hogweed. So the production experience carried out in the Naro-Fominsk urban district of the Moscow region shows that up to 50 hectares can be cultivated in one day. territory littered with Heracleum Sosnowskyi.

Modern remote sensing (RS) technologies make it possible to perform such effective monitoring and obtain information about the state of land resources without direct contact with the object under study.

The specialized RS technology provides spectral analysis and recording of differences of reflected sunlight from the Earth 's surface covered by the studied plants in the state at different stages of their development. Luminance values are combined in certain channels that are informative for highlighting the object under study. They are used to calculate the values of the "spectral index". Based on this data, an image corresponding to a particular index value in each pixel is constructed, which allows you to select the object under study and evaluate its state [3].

The developed technique includes four blocks:

- aerial photography of the territory with the use of UAVs;

- creation of photographic aerial photographs based on materials;

- automated decoding by photoplans of vegetation cover;

- creation of cartograms of distribution areas of Heracleum Sosnowskyi.

The first block. Aerial photography was performed in two zones of the spectrum - red $(\lambda \mathrm{RED}=0.68-0.7 \mu \mathrm{m})$ and near-infrared $(\lambda \mathrm{NIR}=0.74-1.1 \mu \mathrm{m})$. Shooting heights within the routes varied from 250 to $550 \mathrm{~m}$. Longitudinal overlaps of the images were $28 \%$, and transverse $96 \%$ [6]. The second block. Transformation of images was performed using CFS PHOTOMOD. Its digital model with a pitch of $30 \mathrm{~m}$ was used to take into account the terrain. Its construction was carried out on Wednesday GIS "Map 2011". The transformation accuracy ranged from 8 to $10 \mathrm{~m}$.

The third block. The classifier was developed to systematize information about the main plant communities located within the model area. Automated decryption of vegetation cover was performed by comparing for each pixel of the photoplan the indices VI and ClGreen, calculated from dependences (1), (2) with their reference values. Segmentation of plant communities was performed by establishing the affiliation of the selected clusters to the plant community with the subsequent contouring of arrays with the same code $[8,9,10$, 11]. Contour tracking was performed by moving a window along the core boundary along the object's contour with a check at each step of its capture area. As the capture area decreased by more than $10 \%$, the direction of movement of the window changed [7]. For example, Figure 3 shows a fragment of the cartogram of the main types of plant communities in the model area 
In this regard, special field studies are required to obtain spectral characteristics of the physiological state of plants (Heracleum Sosnowskyi), which are obtain on the basis of developed vegetation indices [2]. Spectral characteristics of the plants physiological state allow to determine the parameters necessary for weed control: the phases of plant development.

The spectral characteristics determination of the plants physiological state is based on the field interpretation of remote sensing results covering all phases of development of Heracleum Sosnowskyi in territories with different soil and reclamation conditions and level of water supply. Due to the local dispersal of Heracleum Sosnowskyi remote monitoring of weed vegetation is advisable to carry out unmanned aerial vehicles. The gridding satellite image must be based on well-recognized reference points (at least four) which coordinates are defined. Proposed can be used in other various types of studies [12$15]$.

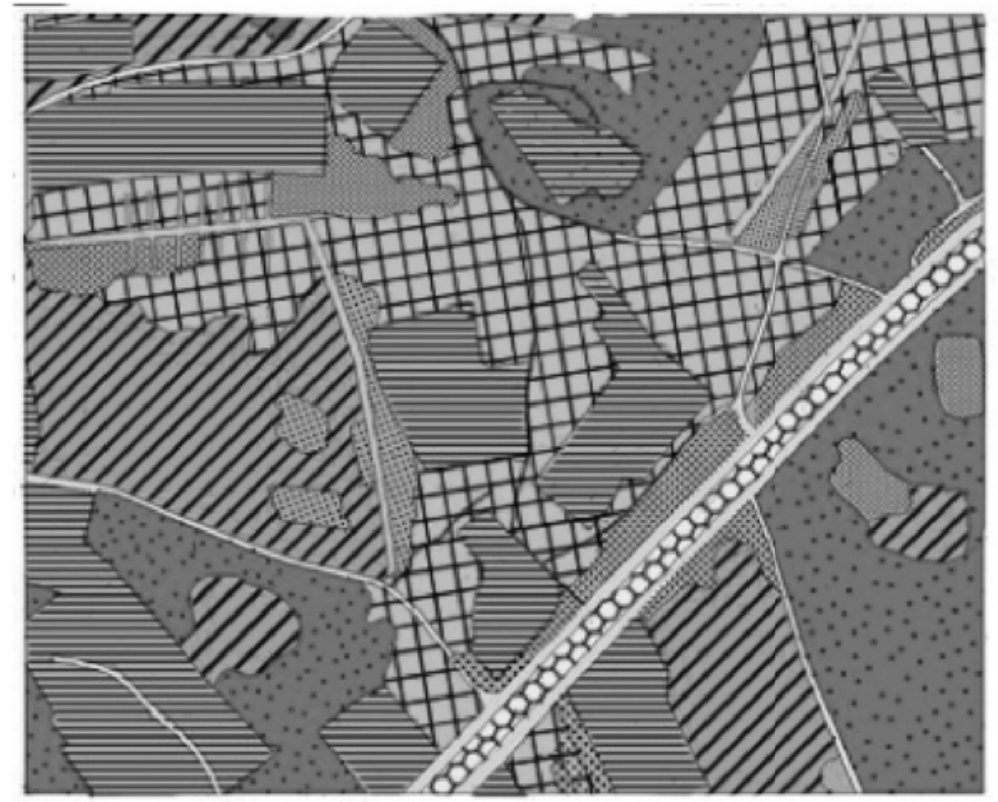

Fig. 3. A fragment of the cartogram of the main types of plant communities in the model area.

\section{Conclusion}

An ecologically dangerous plant of Heracleum Sosnowskyi possesses extremely high reproductive capacity and aggressiveness in the ecosystem, which allows it to freely conquer new territories, forming single-species communities. The resettlement of Heracleum Sosnowskyi in urban and rural areas is a serious environmental and economic problem. The solution of these problems will prevent the risk of chemical burns among the population and introduce large areas of land into economic circulation. Monitoring of territories infested with Heracleum Sosnowskyi using remote sensing means will optimize the technology for the destruction of this weed, reduce the cost of performing technological operations to prevent land contamination with this ecologically dangerous plant. The most effective measures to combat Heracleum Sosnowskyi are the treatment of the territory contaminated with Heracleum Sosnowskyi with herbicides, mulching with polymer film and agrotechnical measures. On lands contaminated with Heracleum Sosnowskyi in urban 
and rural settlements, to reduce the negative impact on the ecosystem, polymeric film mulching and agrotechnical measures should be used to combat hogweed.

The following results were obtained in the course of the performed research:

- A methodology for monitoring land clogging by Heracleum Sosnowskyi has been developed according to remote sensing data, which allows to promptly obtain information on the territorial distribution of clogged lands, their areas and the degree of clogging, which will allow the plan to be improved.

- An unmanned aerial vehicle (UAV) was used to obtain remote sensing data. The survey was performed in two zones of the spectrum - red $(\lambda \operatorname{RED}=0.68-0.7 \mu \mathrm{m})$ and near infrared $(\lambda \mathrm{NIR}=0.74-1.1 \mu \mathrm{m})$.

- Automated decoding of aerial photographs was performed using two vegetation indices: NDVI (NormalizedDifferenceVegetationIndex) and ClGreen (GreenChlorophyllIndex), by comparing the values of the samples for each image.

- NDVI and ClGreen time series were built for five types of plant communities: coniferous forest; deciduous forest; meadows, hayfields, pastures; arable land, vegetable gardens; thickets of Heracleum Sosnowskyi.

- On the basis of the obtained data for the model territory, a sample map of the spatial distribution of the main types of plant communities was constructed, according to which the areas of active growth of Heracleum Sosnowskyi were determined.

- The developed technique can be successfully applied to information support of works at planning of actions on struggle against Heracleum Sosnowskyi. It is expedient to direct further researches on use of the developed technique for creation of electronic maps of distribution of Heracleum Sosnowskyi.

\section{References}

1. V.L. Bogdanov, R.V. Nikolaev, I.V. Shmeleva, Journal of Regional ecology 1-2(31), 43-49 (2011)

2. Supplement to the "Industry classifier of weeds" no. 384021 310. Section: Dicotyledonous perennial roots. Page 37, code 5500. No. 5506- Sosnowsky's hogweed

3. V.P. Yakushev, Materials of the all-Russian scientific conference (with international participation) "The use of remote sensing tools in agriculture" (Saint Petersburg, 2015)

4. V.P. Yakushev, E.V. Kanash, A.A. Konev, SPb AFI 60 (2010)

5. R.A. Poluektov, I.V. Oparina, V.V. Terleev, Russian Meteorology and Hydrology 11, 61-67 (2003)

6. V. Terleev, A. Nikonorov, I. Togo, Y. Volkova, V. Garmanov, D. Shishov, V. Pavlova, N. Semenova, W. Mirschel, Procedia Engineering 165, 1776-1783 (2016)

7. I. Dunaieva, W. Mirschel, V. Popovych, V. Pashtetsky, E. Golovastova, V. Vecherkov, A. Melnichuk, V. Terleev, A. Nikonorov, R. Ginevsky, V. Lazarev, A. Topaj, Advances in Intelligent Systems and Computing 983, 236-246 (2019)

8. A. Nikonorov, V. Badenko, V. Terleev, I. Togo, Y. Volkova, O. Skvortsova, O. Nikonova, S. Pavlov, W. Mirschel, Procedia Engineering 165, 1731-1740 (2016)

9. V.V. Terleev, A.O. Nikonorov, I. Togo, Yu.V. Volkova, R.S. Ginevsky, V.A. Lazarev, E.R. Khamzin, V.V. Garmanov, W. Mirschel, L.I. Akimov, Magazine of Civil Engineering 70(2), 84-92 (2017)

10. V. Terleev, W. Mirschel, A. Nikonorov, V. Lazarev, R. Ginevsky, A. Topaj, K. Moiseev, V. Pashtetsky, I. Dunaieva, V. Popovych, A. Melnichuk, M. Arkhipov, Advances in Intelligent Systems and Computing 983, 462-471 (2019) 
11. V. Terleev, W. Mirschel, A. Nikonorov, R. Ginevsky, V. Lazarev, V. Pavlova, A. Topaj, V. Pashtetsky, I. Dunaieva, V. Popovych, A. Melnichuk, K. Layshev, Advances in Intelligent Systems and Computing 983, 449-461 (2019)

12. T. Orlova, A. Melnichuk, K. Klimenko, V. Vitvitskaya, V. Popovych, I. Dunaieva, V. Terleev, A. Nikonorov, I. Togo, Y. Volkova, W. Mirschel, V. Garmanov, IOP Conference Series: Earth and Environmental Science 90(1), 012110 (2017)

13. R.A. Poluektov, V.V. Terleev, Russian Meteorology and Hydrology 11, 70-75 (2002)

14. V. Terleev, A. Nikonorov, V. Badenko, I. Guseva, Y. Volkova, O. Skvortsova, S. Pavlov, W. Mirschel, Advances in Civil Engineering 2016, 8176728 (2016)

15. V. Terleev, E. Petrovskaia, A. Nikonorov, V. Badenko, Y. Volkova, S. Pavlov, N. Semenova, K. Moiseev, A. Topaj, W. Mirschel, MATEC Web of Conferences 73, 03001 (2016) 